\title{
Comparación en el manejo de pacientes con degeneración mixomatosa mitral con enalapril o con la combinación enalapril-espironolactona
}

\author{
Mauricio Ortega ${ }^{1}$ MV. Esp., Leonardo Gómez² MVZ. Msc. \\ 1,2. Unidad de medicina y cirugía cardiovascular. Programa de Medicina Veterinaria. \\ Universidad Antonio Nariño. Bogotá, Colombia. \\ Correspondencia: mauricio.ortega@uan.edu.co
}

Recibido: 07-02-2007 / Aceptado: 14-03-2007

\begin{abstract}
Resumen
El comportamiento de 45 pacientes caninos con degeneración valvular mixomatosa mitral, manejados con una combinación enalapril- espironolactona (20) o enalapril (25) fueron evaluados en tres ocasiones durante 12 meses, realizando un seguimiento de sobrevivencia, niveles séricos de aldosterona (marcador), creatinina, nitrógeno ureico en sangre, sodio y potasio.

Se observó que la aldosterona tiene una reducción en los niveles séricos posterior al tratamiento con enalapril y espironolactona muy superior a los tratados únicamente con enalapril, pero en la última evaluación, aumentan los niveles por encima de los valores iniciales, lo que sugiere que ya se han activado otras vías diferentes para la síntesis de la aldosterona, que no están mediados por la angiotensina II y que por tal motivo no responden adecuadamente a las enzimas convertidoras de angiotensina. El beneficio de la combinación con espironolactona se basa en que ésta, es un bloqueador selectivo de los receptores para aldosterona, lo que disminuiría su acción letal sobre el miocardio y los vasos sanguíneos.
\end{abstract}

Palabras claves: aldosterona, angiotensina, degeneración valvular mixomatosa, enalapril, falla cardiaca, vasoconstricción.

\begin{abstract}
The behavior of 45 canine patients with degeneration to valvular mixomatosa mitral, handled with a combination enalapril- espironolactona (20) or enalapril (25) were evaluated in three occasions during 12 months, making a pursuit of survival, serum levels of aldosterone (marking), creatinine, ureico nitrogen in blood, sodium and potassium.

It was observed that aldosterone has a reduction in the serum levels subsequent to the treatment with enalapril and espironolactona far beyond the treated solely with enalapril, but in the last evaluation, they increase the levels over the initial values, which suggests they already have activated other routes for the synthesis from the aldosterone, which are not mediated by angiotensin II and therefore they do not respond suitably to converter enzymes of
\end{abstract}


angiotensin. The benefit of the combination with espironolactone is based on its selective blocking a selective of the aldosterone receivers, which would diminish its lethal action on the myocardium and the blood vessels.

Key words: aldosterone, angiotensin, degeneration to valvular myxomatosa, enalapril, fails cardiac, vasoconstriction

\section{Introducción}

En la insuficiencia mitral se presenta una disminución del gasto cardiaco, se activan diversos mecanismos compensatorios neuroendocrinos: el neurogénico mediado por la noradrenalina que es inmediato y a corto tiempo y el endocrino, con la activación del sistema renina-angiotensina-aldosterona (SRAA), que es prolongado en el tiempo, pero tarda en activarse. Este cambio es beneficioso en la primera etapa de la falla cardiaca, pero a largo plazo es la causante de un deterioro del sistema cardiovascular, dado que termina sobrecargando el sistema. Además se ha demostrado que los niveles de aldosterona se elevan en los pacientes con degeneración valvular mixomatosa (1), lo que ocasiona fibrosis del miocardio y de los vasos sanguíneos, así como variabilidad en la frecuencia cardiaca (2).

Es bien sabido, que los pacientes con insuficiencia cardiaca como mecanismo compensatorio, desencadenan una activación neuroendocrina que permite mantener un gasto cardiaco adecuado (3). El SRAA al activarse genera una vasoconstricción vascular periférica debido a la presencia de angiotensina II y un aumento de la volemia mediado por aldosterona al retener líquidos. Se ha demostrado que se presenta un aumento marcado de los niveles de aldosterona sérico en perros con insuficiencia mitral $(1,4)$.

La aldosterona es una hormona que se sintetiza en la glándula adrenal como respuesta a la disminución del flujo renal y en la hiperkalemia. Su efecto permite una regulación hidrosalina mediante la excreción de potasio y la reabsorción de sodio y agua (5). La síntesis de aldosterona depende principalmente de la cascada renina - angiotensina, pero actualmente se sabe que existe una síntesis tisular en el corazón y los vasos sanguíneos que no es dependiente de angiotensina II. El exceso de los niveles de aldosterona causa una fibrosis en el miocardio, variación de la frecuencia cardiaca y sensibilización a las catecolaminas $(2,6)$.

Los tratamientos tradicionales con inhibidores de la enzima convertidora de angiotensina bloquean la síntesis de aldosterona dependiente de este sistema, pero no la ruta tisular. Por lo tanto el efecto nocivo de la aldosterona se perpetúa en la enfermedad cardiaca. El objetivo de esta investigación fue establecer en un grupo de perros con degeneración valvular mixomatosa durante 12 meses, la respuesta a la terapia tradicional con enalapril y a otro grupo, a la terapia con una combinación de enalapril y espironolactona.

\section{Materiales y Métodos}

La población de estudio $(n=45)$ estuvo conformada por pacientes caninos con degeneración valvular mitral, compensada o no compensada, sin importar raza, sexo o edad y sin tratamientos cardiovasculares previos. Estos animales se seleccionaron aleatoriamente para conformar dos grupos: el primero, denominado grupo A con un número de 25 animales, los cuales recibieron como único tratamiento enalapril a una dosis de $0.5 \mathrm{mg} / \mathrm{kg}$ cada 12 horas. El segundo, o grupo B, con un número de 20 animales recibieron como tratamiento la combinación enalapril-espironolactona a una dosis de $0.5 \mathrm{mg} / \mathrm{kg}$ de enalapril y $0.2 \mathrm{mg} / \mathrm{kg}$ de espironolactona, cada 12 horas.

Durante los 12 meses de estudio los animales recibieron manejo habitual en alimentación, condiciones ambientales y ejercicio. En los pacientes en los cuales se presentaron signos congestivos, se medicaron temporalmente con furosemida hasta compensar la 
congestión. Estos pacientes no se retiraron del estudio. A cada uno de los integrantes de los grupos se le practicó el siguiente protocolo de evaluación:

1. Antes de iniciar los estudios, el propietario de cada animal firmó una carta de consentimiento informado, en la cual afirma conocer todas las ventajas y/o desventajas de los tratamientos a seguir y autoriza la realización de todos le exámenes diagnósticos.

2. Se realizó ecocardiograma y electrocardiograma bajo los estándares establecidos, como herramientas diagnósticas complementarias para la clasificación de los pacientes cardiópatas.

3. Se tomó una muestra de sangre por punción en la vena yugular siguiendo las normas de asepsia.
4. Se determinaron los niveles séricos de aldosterona, sodio, potasio, nitrógeno ureico en sangre y creatinina.

5. Mediante oscilometría se determinó la presión arterial.

Este protocolo de evaluación se realizo en 3 ocasiones en cada paciente, al ingresarlo al estudio, en el mes 5 y en el mes 12 después de iniciados los tratamientos. En la segunda evaluación no se determinaron los niveles séricos de sodio y creatinina. En la Figura 1 se muestra las diferentes razas de perros incluidos en el estudio, siendo la población más abundante la raza French Poodle con el $60 \%$ de la muestra, probablemente por ser esta una raza con una alta predisposición a padecer esta patología. En la Figura 2 se presenta las frecuencias por edades de los caninos.

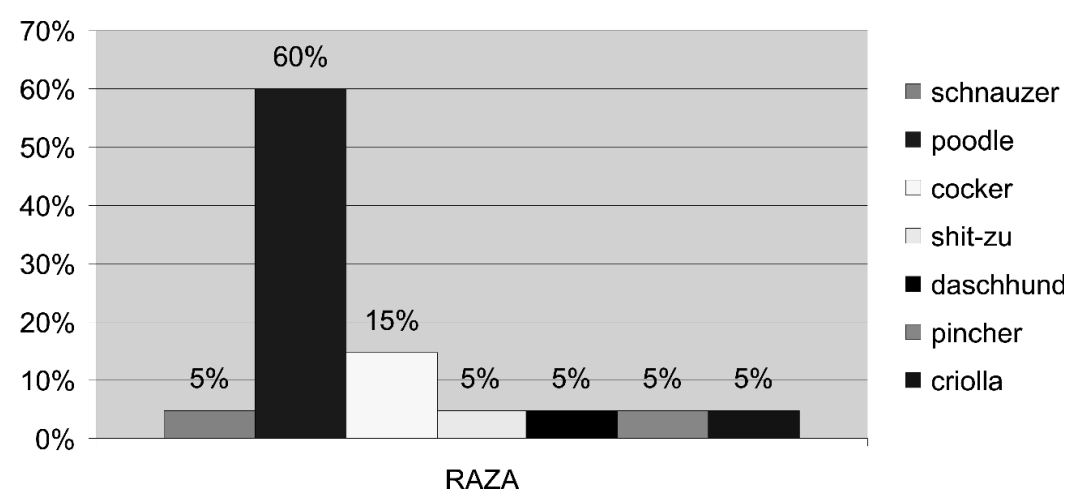

Figura 1. Frecuencia de presentación por razas.

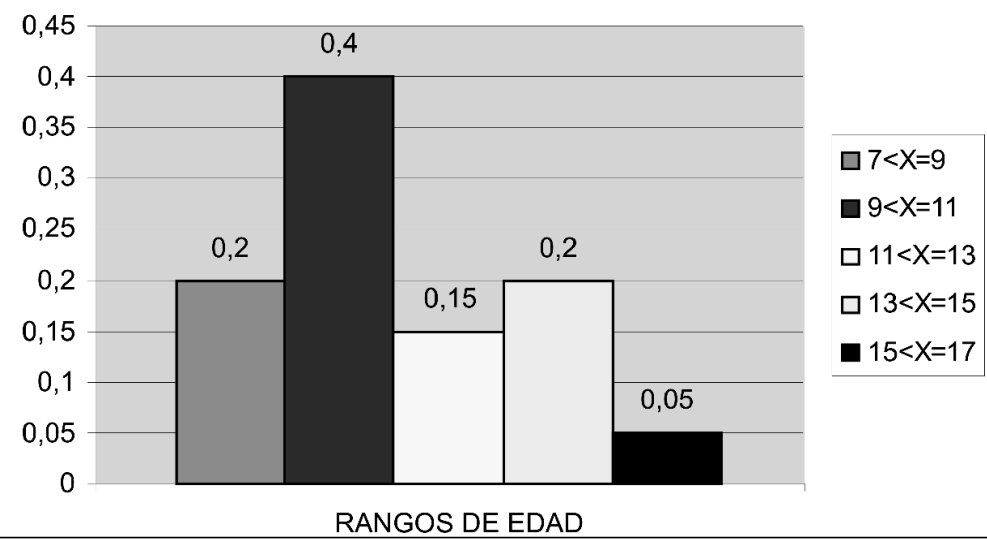

Figura 2. Frecuencia de presentación por edad. 


\section{Resultados}

Se encontró que los pacientes con enfermedad cardiaca tenían una elevación significativa de los valo- res séricos de aldosterona y presiones arteriales medias por encima de los valores de referencia tal y como se muestra en las Tablas 1 y 2 .

Tabla 1. Valoración de 20 pacientes que inician tratamiento con enalapril-espironolactona.

\begin{tabular}{|c|c|c|c|c|}
\hline $\begin{array}{l}\text { MUESTRA (Enalapril } \\
\text { +espironolactona) }\end{array}$ & $\begin{array}{c}\text { VALOR } \\
\text { REFERENCIA }\end{array}$ & MEDIA & MINIMO & MAXIMO \\
\hline Aldosterona (ng/dL) & $5-10$ & 21,236 & 6,09 & 62,11 \\
\hline $\begin{array}{l}\text { Potasio } \\
(\mathrm{mmol} / \mathrm{L})\end{array}$ & $3,8-5,8$ & 4,8684 & 3,7 & 7,1 \\
\hline $\begin{array}{l}\text { Sodio } \\
\text { (mmol/L) }\end{array}$ & $140-158$ & 137,59 & 126 & 169,9 \\
\hline $\begin{array}{l}\text { Creatinina } \\
\text { (mg/dL) }\end{array}$ & $0,5-1,5$ & 1,4 & 0,4 & 2,9 \\
\hline Nitrógeno Ureico (mg/dl) & $10-25$ & 24,62 & 7,4 & 43,3 \\
\hline $\begin{array}{l}\text { Presión arterial media } \\
(\mathrm{mmHg})\end{array}$ & 98,6 & 117,05 & 90 & 151 \\
\hline
\end{tabular}

Tabla 2. Valoración de 25 pacientes que inician tratamiento con enalapril.

\begin{tabular}{lcccc}
\hline MUESTRA ENALAPRIL & $\begin{array}{c}\text { VALOR } \\
\text { REFERENCIA }\end{array}$ & MEDIA & MINIMO & MAXIMO \\
\hline Aldosterona (ng/dL) & $5-10$ & 18,993 & 9,6373 & 42,98 \\
Potasio (mmol/L) & $3,8-5,8$ & 4,716 & 3,6 & 5,8 \\
$\begin{array}{l}\text { Sodio } \\
\text { (mmol/L) }\end{array}$ & $140-158$ & 146,26 & 132,7 & 166,5 \\
Creatinina (mg/dL) & $0,5-1,5$ & 1,352 & & \\
Nitrógeno Ureico & $10-25$ & 19,02 & 6,3 & 2,5 \\
(mg/dL) & & 114,32 & 78,6 & 50,5 \\
Presión Arterial media & 98,6 & & & 132 \\
(mmHg) & & & & \\
\hline
\end{tabular}

En la segunda muestra se observó un descenso importante de los niveles séricos de aldosterona en los dos grupos, pero bastante más marcado en el grupo de enalapril y espironolactona. Estos resultados se presentan en las Tablas 3 y 4.

Tabla 3. Valoración de 12 pacientes que han sobrevivido luego de 5 meses de iniciado el tratamiento con enalapril y espironolactona.

\begin{tabular}{lcccc}
\hline \multicolumn{1}{c}{$\begin{array}{c}\text { MUESTRA enalapril } \\
\quad \text { +espironolactona }\end{array}$} & $\begin{array}{c}\text { VALOR } \\
\text { REFERENCIA }\end{array}$ & MEDIA & MINIMO & MAXIMO \\
$\begin{array}{l}\text { Aldosterona (ng/dL) } \\
\text { Potasio }\end{array}$ & $5-10$ & 12,847 & 7,13 & 27,18 \\
(mmol/L) & $3,8-5,8$ & 5,0677 & 4,33 & 6,07 \\
$\begin{array}{l}\text { Sodio } \\
\text { (mmol/L) }\end{array}$ & $140-158$ & 136,26 & 135,2 & 138 \\
\hline
\end{tabular}

Tabla 4. Valoración de 8 pacientes que han sobrevivido luego de 5 meses de iniciado el tratamiento con enalapril.

\begin{tabular}{lcccc}
\hline MUESTRA ENALAPRIL & $\begin{array}{c}\text { VALOR } \\
\text { REFERENCIA }\end{array}$ & MEDIA & MINIMO & MAXIMO \\
\hline Aldosterona (ng/dL) & $5-10$ & 16,878 & 10,09 & 22,3 \\
Potasio & $3,8-5,8$ & 4,625 & 2,99 & 5,72 \\
(mmol/L) & $140-158$ & 137,22 & 133,7 & 140,1 \\
Sodio & & & & \\
(mmol/L) & & & \\
\hline
\end{tabular}


Para la última muestra, a los doce meses de tratamiento, se observó un leve aumento de los niveles séricos de aldosterona, probablemente debido a la activación de las rutas alternas que no dependen de la angiotensina II. Los datos se presentan en las Tablas 5 y 6.

Tabla 5. Valoración de 8 pacientes que sobrevivieron luego de 12 meses de tratamiento con enalapril y espironolactona.

\begin{tabular}{lcccc}
\hline \multicolumn{1}{c}{$\begin{array}{c}\text { MUESTRA Enalapril } \\
\text { +espironolactona }\end{array}$} & $\begin{array}{c}\text { VALOR } \\
\text { REFERENCIA }\end{array}$ & MEDIA & MINIMO & MAXIMO \\
\hline $\begin{array}{l}\text { Aldosterona (ng/dL) } \\
\text { Potasio }\end{array}$ & $5-10$ & 23,39 & 11,2 & 34,2 \\
(mmol/L) & $3,8-5,8$ & 3,744 & 3,02 & 5,0 \\
$\begin{array}{l}\text { Sodio } \\
\text { (mmol/L) }\end{array}$ & $140-158$ & 140,07 & 132,1 & 145,3 \\
Creatinina (mg/dL) & $0,5-1,5$ & 1,3385 & 0,98 & 1,89 \\
Nitrógeno Ureico (mg/dL) & $10-25$ & 21,66 & 18,5 & 26 \\
\hline
\end{tabular}

Tabla 6. Valoración de 2 pacientes que sobrevivieron luego de 12 meses de tratamiento con enalapril.

\begin{tabular}{lcccc}
\hline MUESTRA ENALAPRIL & $\begin{array}{c}\text { VALOR } \\
\text { REFERENCIA }\end{array}$ & MEDIA & MINIMO & MAXIMO \\
\hline Aldosterona (ng/dL) & $5-10$ & 16,8 & 16,0 & 17,6 \\
Potasio (mmol/L) & $3,8-5,8$ & 4,21 & 4,09 & 4,33 \\
Sodio & $140-159$ & 140,7 & 139,4 & 142,0 \\
(mmol/L) & & & & 1,1 \\
Creatinina (mg/dL) & $0,5-1,5$ & 1,055 & 1,01 & 17,6 \\
Nitrógeno Ureico & $10-25$ & 15,95 & 14,3 & \\
(mg/dL) & & & & \\
\hline
\end{tabular}

\section{Discusión}

Los pacientes con mayor riesgo de muerte súbita, fueron los que tuvieron niveles séricos más elevados de aldosterona; es probable que la fibrosis miocárdica, la sensibilización a las catecolaminas y el efecto proarrítmico de ésta, aumente notoriamente la probabilidad. Esta hormona se activa como un mecanismo de compensación en pacientes con insuficiencia cardiaca, respondiendo a la disminución del flujo renal como consecuencia de un gasto cardiaco disminuido. Esa activación está mediada principalmente por la angiotensina II y se disminuye con el uso de inhibidores de la enzima convertidora de angiotensina (IECA), como se observó en la segunda evaluación de la aldosterona al quinto mes de tratamiento.

En la tercera muestra, realizada en el mes doce de tratamiento, se observa que la aldosterona aumentó nuevamente, pero cabe anotar que dichos aumento fueron menores a los de la primera valoración. La razón para esta dinámica de la aldosterona, es la activación de rutas que no se controlan con los IECA, es decir, que no dependen del SRAA. Como se ha reportado en la literatura, la aldosterona tiene una síntesis que es mediada por el tejido cardiaco y vascular (7).

En este estudio se evidenció que esta reactivación se presentó entre el quinto y décimo mes posterior al uso de tratamientos con IECAS. Este aumento en los niveles séricos de la aldosterona, podría pensarse que producirá menor daño en los pacientes que reciben espironolactona, que como se dijo anteriormente, es un antagonista de la aldosterona por receptores y de esta forma reduce el efecto nocivo sobre el miocardio y sobre la vasculatura cardiaca. De hecho, en el grupo de enalapril y espironolactona sobrevivieron 8 de 20 pacientes, mientras que en el grupo de enalapril, sobrevivieron 2 de 25 pacientes. 
Con los datos encontrados se pueden establecer las siguientes conclusiones:

1. La aldosterona se eleva a largo plazo a pesar del uso de los IECA, por este motivo y conociendo los efectos adversos de esta hormona, sería recomendable por su competencia con receptores aldosterónicos, adicionar fármacos como la espironolactona en pacientes con degeneración valvular mixomatosa mitral.

2. La aldosterona con niveles séricos superiores a 10 $\mathrm{ng} / \mathrm{dl}$, puede ser un importante marcador de probabilidad de muerte súbita en pacientes con degeneración valvular mixomatosa.

3. No se presentaron signos clínicos de falla renal en ninguno de los pacientes, con ninguno de los dos tratamientos establecidos.

4. Los pacientes que demostraron hipertensión arterial en la primera muestra, no lo demostraron en las siguientes dos evaluaciones.
5. Se demostró una sobrevivencia mayor en el grupo manejado con enalapril y espironolactona ( $40 \%$ de los pacientes) comparado con el grupo manejado únicamente con enalapril ( $8 \%$ de los pacientes).

\section{NOVA}

\section{Referencias}

1. Amezquita, K. Romero, A. Determinación y correlación de los niveles de aldosterona sérica con la función ventricular en pacientes caninos cardiópatas y pacientes caninos sanos en la ciudad de Bogotá. Tesis de grado. Universidad de la Salle. 2006.

2. Guyton A. Textbook of Medical Physiology. Ed. 11 Elservier. 2006.

3. Sisson D. Neruoendocrine evaluation of cardiac disease. En: Veterinary Clinics: Small animal practice. Septiembre 2004.

4. Galvan T. Insuficiencia cardiaca: datos para el debate. Panamericana. 2003.

5. Belerenian G. Mucha C. Camacho J. Aparecido A. Afecciones cardiovasculares en pequeños animales. Ed. Intermédica, 2001.

6. Kittleson M. Small animal cardiovascular medicine. Ed. Mosby. 1998.

7. Harrison T. Principios de medicina interna. Mc Graw-HillInteramericana. Ed.14.1998.

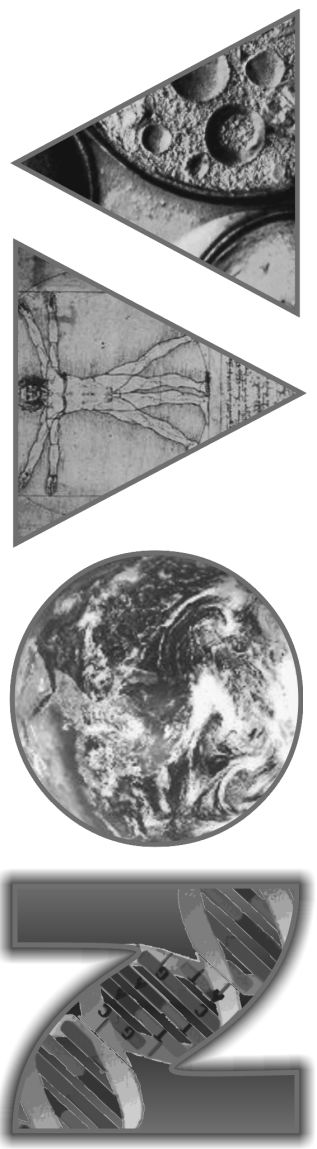

